\title{
Evaluación de polvos de zanahoria obtenidos por deshidratación por aire forzado a diferentes temperaturas
}

\author{
Evaluation of carrot powder obtained by forced-air dehydration \\ at different temperatures
}

\author{
Roxana María Hernández Rendón ${ }^{1 *}$, Danny Javier Blanco Gómez ${ }^{1}$
}

\section{RESUMEN}

El propósito de este trabajo fue la evaluación de polvos de zanahorias deshidratadas aplicando diferentes tratamientos: temperaturas/ tiempo $50{ }^{\circ} \mathrm{C} / 24 \mathrm{~h}, 55{ }^{\circ} \mathrm{C} / 22 \mathrm{~h}$ y $60{ }^{\circ} \mathrm{C} / 20 \mathrm{~h}$. La deshidratación se realizó en una estufa por aire forzado BINDER serie FD 115 (Alemania). Se utilizó un diseño completamente aleatorizado con tres tratamientos (temperaturas/tiempo: $50{ }^{\circ} \mathrm{C} / 24 \mathrm{~h}, 55^{\circ} \mathrm{C} / 22 \mathrm{~h}$ y $60{ }^{\circ} \mathrm{C} / 20 \mathrm{~h}$ ) y cuatro réplicas. Las variables dependientes analizadas fueron los parámetros físicos (aw, color (Índice de Blancura: IB), $\mathrm{pH}$ ), la composición proximal (humedad, ceniza, grasa, proteína, fibra y carbohidratos) y los parámetros nutricionales (carotenos totales y ácido ascórbico) de los polvos de zanahoria obtenidos. Los datos fueron analizados mediante un ANAVA, aplicando un paquete estadístico SAS 2009. Para evaluar la diferencia significativa entre los tratamientos se aplicó una prueba de media de mínima diferencia significativa (m.d.s.) al 5\% de probabilidad. Los resultados del presente estudió mostraron que las temperaturas empleadas no ejercieron ningún efecto sobre la aw ni sobre la composición proximal (humedad, ceniza, grasa, proteínas, fibra y carbohidratos). Los parámetros que se vieron afectados con el incremento de la temperatura de deshidratación fueron: $\mathrm{IB}, \mathrm{pH}$, carotenos totales y ácido ascórbico. El IB se incrementó al aumentar la temperatura de deshidratación mientras que el pH disminuyó. Los carotenos totales y el ácido ascórbico presentaron una mayor degradación al utilizar mayores temperaturas de deshidratación. El polvo de zanahoria obtenido por deshidratación a $50{ }^{\circ} \mathrm{C}$ presentó menor decoloración y mejores características nutricionales por su mayor contenido de carotenos totales y de ácido ascórbico.

Palabras clave: zanahoria, deshidratación, polvo.

\begin{abstract}
The purpose of this study was the evaluation of carrot powder dehydrated at different trearments: temperatures/time $50{ }^{\circ} \mathrm{C} / 24 \mathrm{~h}$, $55^{\circ} \mathrm{C} / 22 \mathrm{~h}$ and $60^{\circ} \mathrm{C} / 20^{\circ} \mathrm{C}$. Dehydration was in forced air oven BINDER FD 115 (Germany). We used a completely randomized design with three treatments (temperatures $50,55,60^{\circ} \mathrm{C}$ ) and four replications. The dependent variables analyzed were parameters physic (aw, color, $\mathrm{pH}$ ), the proximate composition (moisture, ash, fat, protein, carbohydrates and fiber) and nutritional parameters (total carotene, ascorbic acid) of carrot powder obtained. Data were analyzed by ANOVA, using a statistical package SAS 2009. The significant differences between treatments were evaluated applied average test least significant difference $(\mathrm{mds})$ at $5 \%$ probability. The results of this studied showed that the temperatures used had no effect on the aw or on proximate composition (moisture, ash, fat, protein, fiber and carbohydrates). The parameters that were affected by the increased temperature of dehydration were $I B, p H$, total carotenes and ascorbic acid. IB was increased with increasing temperature of dehydration while the $p H$ decreased. Total carotenes and ascorbic acid showed more degradation when using higher temperatures of dehydration. Carrot powder obtained by dehydration at $50^{\circ} \mathrm{C}$ showed less discoloration and better nutritional characteristics by its higher content of total carotenes and ascorbic acid.
\end{abstract}

Key words: carrot, dehydration, powder.

\section{Introducción}

La zanahoria es una raíz fusiforme de la familia Umbelíferas especie Daucus carota L. Es un alimento excelente desde el punto de vista nutricional, ya que es una fuente importante de vitaminas y minerales, y ha sido considerada como uno de los vegetales que presentan los mayores contenidos de fibra dietética natural. El agua es el componente más abundante, seguido de los carbohidratos (nutrientes

\footnotetext{
$1 \quad$ Universidad de Oriente. Departamento Tecnología de Alimentos. Avenida Universidad, Campus Los Guaritos, Universidad de Oriente, Edificio Escuela de Zootecnia. Maturín, Estado Monagas, Venezuela.

* Autor para correspondencia: rhernandez@udo.edu.ve
}

Fecha de Recepción: 4 Febrero, 2015.

Fecha de Aceptación: 14 Agosto, 2015. 
que aportan energía). Su color naranja se debe a la presencia de carotenos, entre ellos el betacaroteno o pro-vitamina A, pigmento natural que el organismo transforma en vitamina A conforme la necesita. Simón y Wolf (1987) señalan que la cantidad de $\beta$-carotenos en zanahoria puede variar de $44 \%$ a $79 \%$ del total de carotenoides. La zanahoria ha tomado un auge importante dentro de la producción mundial de hortalizas, reflejando con ello el amplio gusto que tienen los consumidores por esta. En Venezuela, los cultivos de esta hortaliza se encuentran en las zonas altas, particularmente en la cordillera de Mérida (Mora y Rojas 2007). La zanahoria puede ser consumida cruda, entera o en rebanada, cocida, enlatada, congelada, encurtida o deshidratada. La deshidratación representa una alternativa de procesamiento para esta hortaliza, ya que este proceso estabiliza el producto durante el almacenamiento, además disminuye su peso y volumen, lo que permite disponer de un producto durante todas las épocas del año (Potter y Hotckkiss 1999). La deshidratación de las frutas y vegetales se efectúa usualmente por aire caliente a temperaturas que varían desde $20^{\circ} \mathrm{C}$ hasta $80{ }^{\circ} \mathrm{C}$, sola o en combinación con deshidratación osmótica como pretratamiento (Del Valle et al. 1998). La deshidratación convencional por aire es una de las operaciones usadas más frecuentemente para deshidratar alimento; sin embargo, pueden ocurrir cambios de color significativos y baja capacidad de sorción en el producto final (Krokida et al. 2001). El color es un importante atributo de calidad (Howard et al. 1996), ya que juega un papel importante en la apariencia, procesamiento y aceptación de los productos. Por otra parte, las pérdidas de vitamina A y $\mathrm{C}$ se estudian comúnmente como índice para evaluar la retención de la calidad y el efecto de calentamiento en los métodos de deshidratación (Abonyi et al. 2001). Los alimentos deshidratados pueden ser sometidos a un proceso de molienda para obtener polvos que pueden ser utilizados como ingredientes en la preparación de diversos productos como: jugos, postres, tortas, panes, entre otros. La producción de polvo de zanahoria representa una buena alternativa para aprovechar los importantes beneficios nutricionales que aporta esta raíz. El objetivo fundamental de esta investigación es la evaluación de polvos de zanahoria deshidratados por aire forzado a diferentes temperaturas $50{ }^{\circ} \mathrm{C}$, $55{ }^{\circ} \mathrm{Cy} 60{ }^{\circ} \mathrm{C}$, para ello se determinarán los parámetros físicos (aw, color y $\mathrm{pH}$ ), la composición proximal (humedad, ceniza, grasa, proteína, fibra y carbohidratos) y los parámetros nutricionales (carotenos totales y ácido ascórbico).

\section{Materiales y métodos}

Las raíces de zanahoria variedad Bebe (criollas), 200 unidades, se obtuvieron en estado fresco en un automercado local. Estas fueron lavadas, peladas y cortadas en forma de rodajas de aproximadamente $2 \mathrm{~mm}$ de espesor.

Deshidratación: la deshidratación se realizó en una estufa de aire forzado BINDER serie FD 115 (Alemania), la eficiencia del ventilador se ajustó al $100 \%$. Las temperaturas utilizadas fueron de $50{ }^{\circ} \mathrm{C}, 55^{\circ} \mathrm{C}$ y $60^{\circ} \mathrm{C}$, durante 24,22 y 20 horas. Las muestras fueron retiradas de la estufa y pesadas y se dejaron reposar por 15 minutos en un desecador plástico modelo Vacuum, con el fin de estabilizar su temperatura interna.

Los tiempos de deshidratación se estimaron mediante pruebas preliminares de deshidratación, las muestras fueron retiradas de la estufa y pesadas de hora en hora hasta alcanzar una humedad $\leq 12 \%$.

Molienda: se realizó con un procesador de alimentos marca Oster modelo 3220 pasando el polvo obtenido por un tamiz de $1 \mathrm{~mm}$.

\section{Análisis Físico}

Actividad de Agua (Aw): se realizó utilizando un medidor de Aw AQUALAB DECAGON, Serie 3 (Decagon Devices, Inc. Washington. USA).

pH: se analizó utilizando un medidor de $\mathrm{pH} /$ Temperatura OAKTON, Modelo pH110 (Oakton Instruments. Vernon Hills. USA), según la Norma Venezolana COVENIN 1315-79.

Color: el color de las muestras $\left(L^{*}, a^{*}, b^{*}\right)$ se realizó utilizando un colorímetro COLOR TEC PCM/ PSM (Color Tec Associates, Inc. Clinton, NI) con medida de color en el rango de 400 a $700 \mathrm{~nm}$, sensor $12 \mathrm{~mm}$, cabezal tipo cono portátil, con ventana de zafiro, geometría $45 \%$. Se estimaron los cambios de color de la superficie mediante el índice de blancura (IB, en un rango de 0 al 100), el cual se expresa mediante la fórmula: $\mathrm{IB}=100$ $-\left\{\left(100-\mathrm{L}^{*}\right) 2+\mathrm{a}^{* 2}+\mathrm{b}^{* 2}\right\}^{0,5}$ (Mei et al., 2002).

\section{Composición proximal}

Determinación de humedad: se realizó por el método de calentamiento directo de acuerdo con 
la metodología descrita por la norma venezolana COVENIN 1553-80.

Determinación de cenizas: se realizó por la metodología descrita por la norma venezolana COVENIN 1783-81a.

Determinación de grasa (extracto etéreo): se determinó por el método de Goldfish de extracción continua, usando la metodología descrita por la norma venezolana COVENIN 1785-81b.

Determinación de proteína: se realizó por el método de Microkjeldahl, usando la metodología descrita por la norma venezolana COVENIN 1195-95.

Determinación de fibra cruda: se realizó usando la metodología descrita por la norma venezolana COVENIN 1789-81c.

Determinación de carbohidratos: se obtuvo por diferencia, aplicando la siguiente fórmula (FAO 1996): \% Carbohidratos $=100-(\%$ humedad + $\%$ grasa $+\%$ proteínas $+\%$ fibra $+\%$ cenizas $)$.

\section{Análisis nutricional}

Contenido de ácido ascórbico (AA): se determinó mediante el método de volumetría de óxido-reducción, titulando con el colorante 2,6 dicloroindofenol, siguiendo la metodología empleada por Zago et al. (2010).

Contenido de carotenos totales: las concentraciones de carotenos totales se determinó por espectrofotometría a $450 \mathrm{~nm}$, utilizando un espectrofotómetro SPECTRONIC 20 Génesis modelo $10 U V$, de Spectronic Instruments Inc. La extracción de carotenos totales en fase orgánica se realizó de acuerdo con el método descrito por Brittón y Hornero (1997).

\section{Diseño experimental}

Se utilizó un diseño completamente aleatorizado con tres tratamientos (temperaturas/tiempo: T1 $50{ }^{\circ} \mathrm{C} / 24 \mathrm{~h}, \mathrm{~T} 255^{\circ} \mathrm{C} / 22 \mathrm{~h}$ y T3 $60{ }^{\circ} \mathrm{C} / 20 \mathrm{~h}$ ) y cuatro réplicas. Las variables dependientes analizadas fueron los parámetros físicos (aw, color y $\mathrm{pH}$ ), la composición proximal (humedad, ceniza, grasa, proteína, fibra y carbohidratos) y los parámetros nutricionales (carotenos totales y ácido ascórbico) de los polvos de zanahoria obtenidos.

\section{Análisis estadístico}

Los datos fueron analizados mediante un análisis de varianza. Para evaluar la diferencia significativa entre los tratamientos se aplicó una prueba de media de mínima diferencia significativa (m.d.s.) al 5\% de probabilidad. Para el análisis de los datos se utilizó el paquete estadístico computarizado SAS 2009.

Localización de la investigación. La investigación se realizó en el Laboratorio de Tecnología de los Alimentos y el Laboratorio de Nutrición Animal y Forraje de la Universidad de Oriente, Núcleo de Monagas, Venezuela y en el Laboratorio Fitopatología del Instituto Nacional de Investigaciones Agrícolas (INIA), Monagas, Venezuela.

\section{Resultados y discusión}

\section{Análisis físicos de los polvos de zanahorias deshidratadas a diferentes temperaturas}

En la Tabla 1 se presentan los resultados de los análisis físicos realizados a los polvos de zanahorias deshidratadas aplicando diferentes tratamientos (temperaturas/tiempos).

Actividad de agua (aw): los valores de aw obtenidos no mostraron diferencias estadísticamente significativas entre los tratamientos $(\mathrm{p}<, 05)$ (Tabla 1). Esto indica que los tratamientos no ejercieron ningún efecto significativo sobre este parámetro. Los valores de aw estuvieron comprendidos entre 0,295 y 0,313 . Los productos con aw menores a 0,6 se consideran de baja humedad, en estos no hay crecimiento bacteriano y el pardeamiento enzimático disminuye (Potter y Hotchkiss 1999), lo cual conlleva aumentar el tiempo de vida útil del producto.

Color (Índice de blancura, IB): existe diferencia significativa entre los tratamientos, lo que indica que los tratamientos aplicados afectaron este parámetro. Los valores de IB se presentan en la Tabla 1. Se puede observar que a mayor temperatura

Tabla 1. Valores de los análisis físicos del polvo de zanahorias deshidratadas aplicando diferentes tratamientos

\begin{tabular}{lccc}
\hline \multirow{2}{*}{ Análisis físico } & \multicolumn{3}{c}{ Tratamientos } \\
\cline { 2 - 4 } & $\mathrm{T} 1: 50^{\circ} \mathrm{C} / 24 \mathrm{~h}$ & $\mathrm{~T} 2: 55^{\circ} \mathrm{C} / 22 \mathrm{~h}$ & $\mathrm{~T} 3: 60^{\circ} \mathrm{C} / 20 \mathrm{~h}$ \\
\hline Aw & $0,313^{\mathrm{a}}$ & $0,311^{\mathrm{a}}$ & $0,295^{\mathrm{a}}$ \\
Índice de & & & \\
blancura (IB) & $36,924^{\mathrm{a}}$ & $39,837^{\mathrm{b}}$ & $42,755^{\mathrm{c}}$ \\
$\mathrm{pH}$ & $6,0062^{\mathrm{a}}$ & $5,6825^{\mathrm{b}}$ & $5,3282^{\mathrm{c}}$ \\
\hline
\end{tabular}

Los valores son promedios de cuatro repeticiones

a, b, c, Medias con letras diferentes dentro de la misma fila son significativamente diferentes $(\mathrm{p}<0,05)$. 
y menor tiempo se incrementa el IB, lo que conlleva a que el producto ha sufrido una mayor decoloración por efecto de la temperatura de deshidratación. Mei et al. (2002) señala que el valor más alto de IB representa la mayor decoloración de la superficie. Por otra parte, Uquire y Cisneros (2002) mencionan que cuando el índice de blancura se encuentra entre valores de 31 y 37 , generalmente no se ve formación blanca en la superficie de la zanahoria, pero sí un cambio de color de naranja intenso a naranja pálido, lo que se observó en el tratamiento $50{ }^{\circ} \mathrm{C} / 24 \mathrm{~h}$. El tratamiento que presentó el menor IB fue $50{ }^{\circ} \mathrm{C} / 24$ h, el cual permite conservar el color en polvo de zanahoria en comparación con los otros tratamientos $\left(55^{\circ} \mathrm{C} / 22 \mathrm{~h}\right.$ y $60{ }^{\circ} \mathrm{C} / 20 \mathrm{~h}$ ) estudiados.

pH: los valores de $\mathrm{pH}$ de los polvos de zanahorias obtenidos aplicando diferentes tratamientos $\left(50^{\circ} \mathrm{C} / 24\right.$ $\mathrm{h}, 55{ }^{\circ} \mathrm{C} / 22 \mathrm{~h}$ y $\left.60{ }^{\circ} \mathrm{C} / 20 \mathrm{~h}\right)$, se muestran en la Tabla 1. Se encontró diferencia significativa $(\mathrm{p}<$ 0,05 ) entre todos los tratamientos observándose que a medida que se incrementó la temperatura y disminuyó el tiempo de deshidratación, el pH disminuyó, es decir, se tornó más ácido. Dotro et al. (1997) señalan que a medida que la temperatura aumenta, el pKa de los ácidos disminuye, por tanto, a mayor temperatura sus características químicas cambian, haciendo mucho más fácil su disociación y de esta manera más ácida la solución produciendo el efecto inmediato de la disminución del $\mathrm{pH}$.

\section{Composición proximal de los polvos de zanahorias deshidratadas a diferentes temperaturas}

La composición proximal de los polvos de zanahorias deshidratados a $50{ }^{\circ} \mathrm{C}, 55^{\circ} \mathrm{C}$ y $60{ }^{\circ} \mathrm{C}$ se presentan en la Tabla 2. Los valores obtenidos para humedad, cenizas, grasa, proteína, fibra y carbohidratos no presentaron diferencias estadísticas significativas $(\mathrm{p}<0,05)$, lo que nos indica que los tratamientos en estudio no afectaron a ninguno de estos parámetros.

Contenido de humedad: el porcentaje de humedad obtenido se encontró entre 10,65\% y $11,36 \%$. Holdsworth (1998) y Parsons (1992) señalan que la humedad para alimentos en polvo debe estar comprendida entre $12 \%$ y $15 \%$.

Contenido de cenizas: los valores de cenizas para los polvos de zanahoria se encuentran entre $7,62 \%$ y $8,75 \%$. Bao y Chang (1994) reportaron para pulpa de zanahoria valores de 5 a $6 \%$ de minerales.
Contenido de proteína: los valores obtenidos en este parámetro se encuentran entre 7,95\% y $8,85 \%$. Bao y Chang (1994) reportaron para pulpa de zanahoria valores de $4 \%$ a $5 \%$ proteínas.

Contenido de grasa (extracto etéreo): se obtuvieron valores de 1,30\% a 1,4\%. El contenido de grasas en los polvos de zanahorias obtenidos es bajo, un mayor contenido pudiera afectar la conservación del mismo, ya que se produciría enranciamiento.

Contenido de fibra cruda: los valores de fibra cruda obtenidos en los polvos de zanahorias estuvieron entre $7,80 \%$ y $7,89 \%$. El polvo de zanahoria puede ser una buena fuente de fibra en la dieta.

Contenido de carbohidratos: Se obtuvieron valores de carbodridratos entre $62,64 \%$ y $63,70 \%$. Los carbohidratos es el macronutriente principal contenido en los polvos de zanahoria.

\section{Análisis nutricionales de los polvos de zanahorias deshidratadas a diferentes temperaturas}

En la Tabla 3 se presentan las variables nutricionales realizadas a los polvos de zanahorias, sometidas a los diferentes tratamientos.

Tabla 2. Composición proximal de los polvos de zanahorias deshidratadas por aire forzado a diferentes temperaturas.

\begin{tabular}{lccc}
\hline \multirow{2}{*}{$\begin{array}{l}\text { Composición } \\
\text { proximal }\end{array}$} & \multicolumn{3}{c}{ Tratamientos } \\
\cline { 2 - 4 } & $\mathrm{T} 1: 50^{\circ} \mathrm{C} / 24 \mathrm{~h}$ & $\mathrm{~T} 2: 55^{\circ} \mathrm{C} / 22 \mathrm{~h}$ & $\mathrm{~T} 3: 60^{\circ} \mathrm{C} / 20 \mathrm{~h}$ \\
\hline Humedad (\%) & $11,36^{\mathrm{a}}$ & $10,93^{\mathrm{a}}$ & $10,65^{\mathrm{a}}$ \\
Cenizas (\%) & $7,62^{\mathrm{a}}$ & $8,17^{\mathrm{a}}$ & $8,75^{\mathrm{a}}$ \\
Grasa (\%) & $1,42^{\mathrm{a}}$ & $1,31^{\mathrm{a}}$ & $1,30^{\mathrm{a}}$ \\
Proteína (\%) & $7,95^{\mathrm{a}}$ & $8,28^{\mathrm{a}}$ & $8,85^{\mathrm{a}}$ \\
Fibra (\%) & $7,89^{\mathrm{a}}$ & $7,83^{\mathrm{a}}$ & $7,80^{\mathrm{a}}$ \\
Carbohidratos (\%) & $63,70^{\mathrm{a}}$ & $63,47^{\mathrm{a}}$ & $62,64^{\mathrm{a}}$ \\
\hline
\end{tabular}

Los valores son promedios de cuatro repeticiones

a, b, c, Medias con letras diferentes dentro de la misma fila son significativamente diferentes $(\mathrm{p}<0,05)$.

Tabla 3. Valores de los parámetros nutricionales de polvos de zanahorias deshidratadas por aire forzado a diferentes temperaturas.

\begin{tabular}{lccc}
\hline \multirow{2}{*}{ Análisis nutricional } & \multicolumn{3}{c}{ Tratamientos } \\
\cline { 2 - 4 } & $\mathrm{T} 1: 50^{\circ} \mathrm{C} / 24 \mathrm{~h}$ & $\mathrm{~T} 2: 55^{\circ} \mathrm{C} / 22 \mathrm{~h}$ & $\mathrm{~T} 3: 60^{\circ} \mathrm{C} / 20 \mathrm{~h}$ \\
\hline $\begin{array}{l}\text { Carotenos totales } \\
(\mathrm{mg} / 100 \mathrm{~g})\end{array}$ & $33,842^{\mathrm{a}}$ & $27,796^{\mathrm{b}}$ & $21,611^{\mathrm{c}}$ \\
$\begin{array}{l}\text { Ácido ascórbico } \\
(\mathrm{mg} \mathrm{AA} / 100 \mathrm{~g})\end{array}$ & $18,443^{\mathrm{a}}$ & $10,824^{\mathrm{b}}$ & $4,782^{\mathrm{c}}$ \\
\hline
\end{tabular}

Los valores son promedios de cuatro repeticiones

a, b, c, Medias con letras diferentes dentro de la misma fila son significativamente diferentes $(\mathrm{p}<0,05)$. 
Contenido de carotenos totales: Se observaron diferencias estadísticas significativas $(\mathrm{p}<0,05)$ entre los tratamientos (Tabla 3), indicando que los tratamientos aplicados ejercen un efecto sobre el contenido de carotenos totales. Los mayores valores de este parámetro lo presentaron los polvos de zanahorias deshidratadas a $50^{\circ} \mathrm{C} / 24 \mathrm{~h}$, disminuyendo el contenido a medida que se incrementaba la temperatura. Gamboa et al. (2007) señalan que en panquecas elaboradas a base de harina de trigo y zanahoria las mezclas crudas tienen mayor contenido de carotenoides que la mezcla cocida. Esto permite inferir que el tratamiento térmico permite la degradación de los carotenos y que a medida que se incrementa la temperatura se produce una mayor pérdida de estos. Los cubos de zanahorias escaldados y deshidratados podrían representar una buena fuente de $\beta$-caroteno en la dieta.

Contenido de ácido ascórbico: los resultados obtenidos de ácido ascórbico en los polvos de zanahorias deshidratadas a tres temperaturas se encuentran en la Tabla 3. Se encontró diferencia significativa $(p<0,05)$ entre todos los tratamientos, lo que indica que los tratamientos estudiados afectan el contenido de ácido ascórbico en los polvos de zanahorias obtenidos. En el estudio realizado por
Alasalvar et al. (2001) se observó que zanahorias frescas de variedad naranjas contenían $5,33 \mathrm{mg}$ $\mathrm{AA} / 100 \mathrm{~g}$, estos valores son muy parecidos a los obtenidos en el polvo de zanahorias deshidratadas a $60{ }^{\circ} \mathrm{C} / 20 \mathrm{~h}$. Para los tratamientos de $50{ }^{\circ} \mathrm{C} / 24 \mathrm{~h}$ y $55^{\circ} \mathrm{C} / 22 \mathrm{~h}$ el contenido de ácido ascórbico fue mayor que en la zanahoria fresca, esto se debe a que durante la deshidratación se pierde agua y se concentran los componentes del alimento.

\section{Conclusiones}

Los tratamientos no ejercieron ningún efecto sobre la aw ni sobre la composición proximal (humedad, cenizas, grasa, proteínas, fibra y carbohidratos). Los parámetros que se vieron afectados por el proceso de deshidratación fueron: $\mathrm{IB}, \mathrm{pH}$, carotenos totales y ácido ascórbico. El IB se incrementó al aumentar la temperatura de deshidratación mientras que el pH disminuyó. Los carotenos totales y el ácido ascórbico presentaron una mayor degradación al utilizar mayores temperaturas de deshidratación. El polvo de zanahoria obtenido por deshidratación a $50{ }^{\circ} \mathrm{C}$ presentó menor decoloración y mejores características nutricionales por su mayor contenido de carotenos totales y de ácido ascórbico.

\section{Literatura Citada}

Abonyi, B.; Feng, H.; Tang, J.; Edwads, C.; Chew, B.; Mattinson, D.; Fellman, J.

2001. Quality retencion in strawberry and carrot purees dried with refractance windowTM system. Journal of Food Science, 67 (2): 1051-1056.

Alasalvar, C.; Grigor, J.; Zhang, D.; Peter, C.; Shahidi, F. 2001 Comparason of volátiles, phenolics, sugar, antioxidant vitamins, and sensory quality of different colored carrot varieties. J. Agric Food Chen, 49: 1410-1416.

Bao. B.; Chang, K.

1994. Carrot pulp chemical composition, color, and waterholding capacity as affected by blanching. Journal of Food Science, 59 (6): 1159-1161.

Brittón, G.; Hornero, M.

1997. Carotenoids and Colour in Fruit and Vegetables", In: Phytochemistry of Fruit and Vegetables, Tomás-Barberán, F. A. and Robins, R. J, eds, Clarendon Press, Oxford, pp. 11-27.

COVENIN

1977. Comisión Venezolana de Normas Industriales. Alimentos. Determinación del pH (Acidez Iónica). Norma Venezolana 1151-77. FONDONORMA, Caracas, Venezuela. 7 pp.

COVENIN. Comisión Venezolana de Normas Industriales. 1980. Frutas y productos derivados. Determinación de humedad. Norma Venezolana 1553-80. FONDONORMA, Caracas, Venezuela. $6 \mathrm{pp}$.
COVENIN. Comisión Venezolana de Normas Industriales. 1981a. Alimentos: Determinación de Cenizas. 1783-81. $1^{\text {a }}$ Revisión. FONDONORMA, Caracas, Venezuela. 6 pp. COVENIN. Comisión Venezolana de Normas Industriales. 1981b. Alimentos: Determinación de Grasa.1785-81. $1^{\text {a }}$ Revisión. FONDONORMA, Caracas, Venezuela. 7 pp. COVENIN. Comisión Venezolana de Normas Industriales. 1981c. Alimentos: Determinación de Fibra Cruda. 1789-81. $1^{\mathrm{a}}$ Revisión. FONDONORMA, Caracas, Venezuela. 6 pp. COVENIN. Comisión Venezolana de Normas Industriales. 1995. Alimentos: Determinación de Proteínas. 1195-95. $1^{\mathrm{a}}$ Revisión. FONDONORMA, Caracas, Venezuela. 10 pp.

Del Valle, J.; Cuadros, T.; Aguilera, J. 1998. Glass transitions and ahrinkage during drying and storage of osmonsed apple. Food research International, 31 (3): 191-204.

Dotro P, Nardi M, Rodríguez D, Rodríguez, V.

1997. Estudio de la Evolución del pH en Función de la Temperatura, Club de Ciencias "Leonardo Da Vinci" Departamento de Investigación y Desarrollo, E.M.E.T. No4, D.E. XVIII, "Hipólito Yrigoyen", Ciudad de Buenos Aires, Argentina. [Documento en línea]. Disponible en: http://www.elysium.com.ar/science/ phys/files/ph.pdf. 65 pp. Consultado: Mayo, 2010.

Gamboa, L.; González, M.; Hurtado, E.

2007. Valoración nutricional y sensorial de panquecas elaboradas a base de harina de trigo (Triticum aestivum $\mathrm{L}$ ) y zanahoria (Daucus carota L). Idesia, 25 (1): 47-52. 
Holdsworth, S.

1998. Conservación de frutas y hortalizas, Acribia, España, $186 \mathrm{pp}$.

Howard, L.; Braswell, D.; Aselage, J.

1996. Chemical composition and color of strained carrots as affected by processing. Journal of Food Science, 61 (2): 327-330.

Krokida, M.; Maroulis, Z.; Saracavos, G.

2001. The effect of the method of drying on the colour of dehydrated products. International J Food Sci and Tech., 36: 53-59.

Mei Y, Zhao, J Yang J, Furr. H.

2002. Using edible coating to enchance nutritional and sensory qualities of baby carrots. J Food Sci 67 (65): 1964-1968.

Mora, E.; Rojas, J.

2007. Los cultivos líderes de la agricultura venezolana (19842005). Agroalim, 12 (25): 33-44.
Parsons, D.

1992. Manuales de educación agropecuaria: Curcubitaceaes, Acribia, Zaragoza. España, 56 pp.

Potter N, Hotchkiss J.

1999. Ciencia de los alimentos. $5^{\text {a }}$ Ed, Acribia, Zaragoza, España. $221 \mathrm{pp}$.

Simón P, Wolff X.

1987. Carotenes in typical and dark orange carrots. J. Agric. Food Chem., 35:1017-1022.

Uquire, E.; Cisneros, L.

2002. Efecto del escaldado y recubrimiento higroscópico sobre la calidad de zanahorias (Daucus carota var. Chantenay) precortadas durante el almacenamiento. ALAN, 52 (2): 187-192.

Zago G, Karina I, García F, María Y, Bernardo D, María L.

2010. Determinación del contenido de vitamina $\mathrm{C}$ en miel de abejas venezolanas por volumetría de óxido-reducción. INHRR, 41 (1): 25-30. 